\title{
Forecasting Seismic Signatures of Stellar Magnetic Activity
}

\author{
W. A. Dziembowski \\ Warsaw University Observatory, Aleje Ujazdowskie 4, 00-478 and Copernicus Astronomical \\ Center, ul. Bartycka 18, 00-716 Warszawa, Poland
}

\begin{abstract}
For the Sun, a tight correlation between various activity measures and oscillation frequencies is well documented. For other stars, we have abundant data on magnetic activity and its changes but not yet on its seismic signature. A prediction of the activity induced frequency changes in stars based on scaling the solar relations is presented. This seismic signature of the activity should be measurable in the data expected within few years.
\end{abstract}

Keywords: Helioseismology, solar activity, stellar activity, stellar seismology, $\beta$ Hyi

PACS: 96.60.Ly, 96.60.qd, 97.10.Jb, 97.10.Sj, 97.20.-w

\section{INTRODUCTION}

Only a few years after the discovery of low-degree modes of the solar five minute oscillations, Woodard and Noyes (1985) showed using data from the SMM mission taken during the declining phase of cycle 21 that the oscillation frequencies decrease with decreasing Sun's activity. The correlation between various activity measures and the frequencies was definitely confirmed with the data taken during the next activity cycle (Libbrecht \& Woodard 1990; Bachmann \& Brown 1993; Regulo et al. 1994; Elsworth et al. 1994). High precision frequency measurements are now available for a number of solar-like stars (e.g. Bedding \& Kjeldsen 2007). Although, the expected seismic signature of the magnetic activity is very weak indeed, its discovery seems to be only a matter of time.

The relative frequency changes in the eleven-year cycle are at most of the order of $10^{-4}$, which is one order of magnitude less than the changes in the irradiance and three orders less than in the chromospheric emission measures. We should expect a similar situation in distant stars. For many solar-like objects, cyclic variations variations of the chromospheric emission are well established (e.g. Baliunas 1998). Nonetheless, an effort to measure the much weaker seismic signature is of interest because it reflects the changes taking place in deeper layers.

In expectation of data from measurements, Metcalf et al. (2007, hereinafter M07) developed a method for predicting frequency shifts based on scaling the measured pmode frequency variations and changes of the chromospheric activity indices in the Sun. Specific forecast was made for the radial modes in the subgiant $\beta$ Hyi. In this paper, I outline the principle of the forecasting method adopted in that work and expand on the part concerning nonradial modes. 


\section{ACTIVITY RELATED CHANGES IN OSCILLATION FREQUENCIES}

The starting point in the forecast presented in M07 was the variational expression for frequency shifts

$$
\Delta v_{j}=\frac{\int d^{3} x \mathscr{K}_{j} \mathscr{S}}{2 I_{j} v_{j}},
$$

where $j \equiv(n, \ell, m)$ identifies the mode, $\mathscr{K}_{j}$ is the kernel, which depends on the unperturbed stellar model parameters and eigenvectors of its free oscillations, $\boldsymbol{\xi}(\boldsymbol{x})$. The quantity

$$
I_{j}=\int d^{3} \boldsymbol{x} \rho|\boldsymbol{\xi}|^{3}=R^{5} \bar{\rho} \tilde{I}
$$

denotes the mode inertia. The second equality defines the dimensionless mode inertia, $\tilde{I}$. The source, $\mathscr{S}$, must include the direct feed-back from the magnetic field perturbed by an oscillation mode but also the indirect field effects caused by the perturbation of the equilibrium density, temperature, and velocity of convection. All these effects may be important for frequency shifts at a level of $0.1 \mu \mathrm{Hz}$. Disentangling these effects in the solar data is impossible and calculation of their relative contributions to the source is still very uncertain. The general form of the kernels for various effects (see e.g. Goldreich et al. 1991, Dziembowski \& Goode 2004) is complicated but for all of them the leading terms are proportional to $\left|\operatorname{div} \xi_{j}\right|^{2}$.

In their semi-empirical determination of $\mathscr{S}(\boldsymbol{x})$ for the Sun, M07 adopted the common kernel

$$
\mathscr{K}_{j}(\boldsymbol{x})=\left|\operatorname{div} \boldsymbol{\xi}_{j}\right|^{2}=q_{j}(D)\left|Y_{\ell}^{m}\right|^{2},
$$

where $D$ denotes the depth below the photosphere, absorbing the model dependent parameters such as gas density or adiabatic exponent in the unknown source $\mathscr{S}$. Only the part of $\mathscr{S}$ that is symmetric about the equator affects mode frequencies. Therefore, without loss of generality, they could use the following expression

$$
\mathscr{S}(\boldsymbol{x})=\sum_{k=0} \mathscr{S}_{k}(D) P_{2 k}(\mu)
$$

where $\mu=\cos \theta$. It follows from equations (1), (3), and (4) that only the terms up to $k=\ell$ contribute to the shifts of modes with degree $\ell$.

\section{FREQUENCY CHANGES IN THE SUN}

Most of what we know about the changes in the solar oscillation frequencies comes from disc-resolved measurements. The data from such measurements are now commonly represented in the form

$$
v_{n l m}=\sum_{i=0} a_{i, n \ell} \mathscr{P}_{i}^{\ell}(m)
$$

where $\mathscr{P}_{i}^{\ell}(m)$ are orthogonal polynomials of order $i$ (see e.g. Schou et al. 1994). The mean frequencies, $a_{0}$, yield information on the radial structure of the Sun. The 
higher even-order $a_{k}$-coefficients yield separately information on the Sun's asphericity described by the individual Legendre polynomial $P_{2 k}(\mu)$. Thus, from measured shifts, $\Delta a_{2 i}(i=0,1, \ldots)$, as functions of the mean frequency, we get our constraints on $\mathscr{S}_{2 i}(D)$ in the Sun.

Using equations (3) and (4) in (1), we get

$$
\Delta v_{n l m}=\frac{q_{n l} \sum_{k=0}^{\ell} \mathscr{S}_{k} \kappa_{k, l m}}{2 I_{n l} v_{n l}}
$$

where,

$$
\kappa_{k, l m} \equiv \int d \mu d \phi\left|Y_{\ell}^{m}\right|^{2} P_{2 k}(\mu)=\mathscr{P}_{2 k}^{\ell}(m) Z_{k}^{\ell}
$$

and

$$
Z_{k}^{\ell}=(-1)^{k} \frac{(2 k-1) ! !}{k !} \frac{(2 \ell+1) ! !}{(2 \ell+2 k+1) ! !} \frac{(\ell-1) !}{(\ell-k) !} .
$$

This equation with $\mathscr{S}_{k}$ derived from data extending from $\ell=0$ to over $\ell=100$, allows us to determine the shifts of the low- $\ell$ modes much more accurately than if they may have been measured directly.

Data on the frequency shifts show that the source is localized near the Sun's photosphere. Thus, the following simple form of the depth dependence of the coefficients in (4) was assumed in M07:

$$
\mathscr{S}_{k}(D)=1.5 \times 10^{-11} A_{k} \delta\left(D-D_{c, k}\right) \mu \mathrm{Hz}^{2},
$$

where $A_{k}$ and $D_{c, k}$ were determined by fitting equation (7) to the data of the $a_{2 k}$ coefficient shifts, using the relation

$$
\Delta a_{2 k, n \ell}=A_{k} Z_{k, \ell} Q_{n \ell}\left(D_{c, k}\right),
$$

where we denoted

$$
Q_{n \ell}=1.5 \times 10^{-11} \frac{q_{n \ell}}{v_{n \ell} \tilde{I}_{n \ell}} .
$$

The relation (8) follows from equations (5), (6), (7), and (2). The numerical factor is arbitrary. M07 determined only the value of $A_{0}$, using data for p-modes with $n>1$ and $v>2.5$. They showed that the mean frequency shifts, $\Delta a_{0}$, for over $1500 \mathrm{p}$-modes are well described with our two-parameter fit and that $D_{c, 0} \approx 0.3 \mathrm{Mm}$. There was a small systematic departure at low frequencies, indicating that the source extends beneath 0.3 $\mathrm{Mm}$, but it was regarded to be unimportant. For other $a_{2 k}$ of low $k$, the fit is also good at similar values of $D_{c, k}$. Thus, data on shifts for each of the low order even $a$-coefficients may be reduced to the single parameters $A_{k}$. How these parameters correlate with the MgII activity index is shown in Figure 1 . The plotted $A_{k}$ values were determined on the basis of the $a_{2 k}$ coefficients calculated by Jesper Schou for the SOHO MDI data1. The values of $\Delta i_{\mathrm{MgII}}$ shown in the bottom panel are from M07 and they were based on the NOAA data2.

\footnotetext{
${ }^{1} \mathrm{http}: / / q u a k e . S t a n f o r d . E D U /$ schou/asym1/

2 http://www.sec.noaa.gov/ftpdir/sbuv/NOAMgII.dat
} 

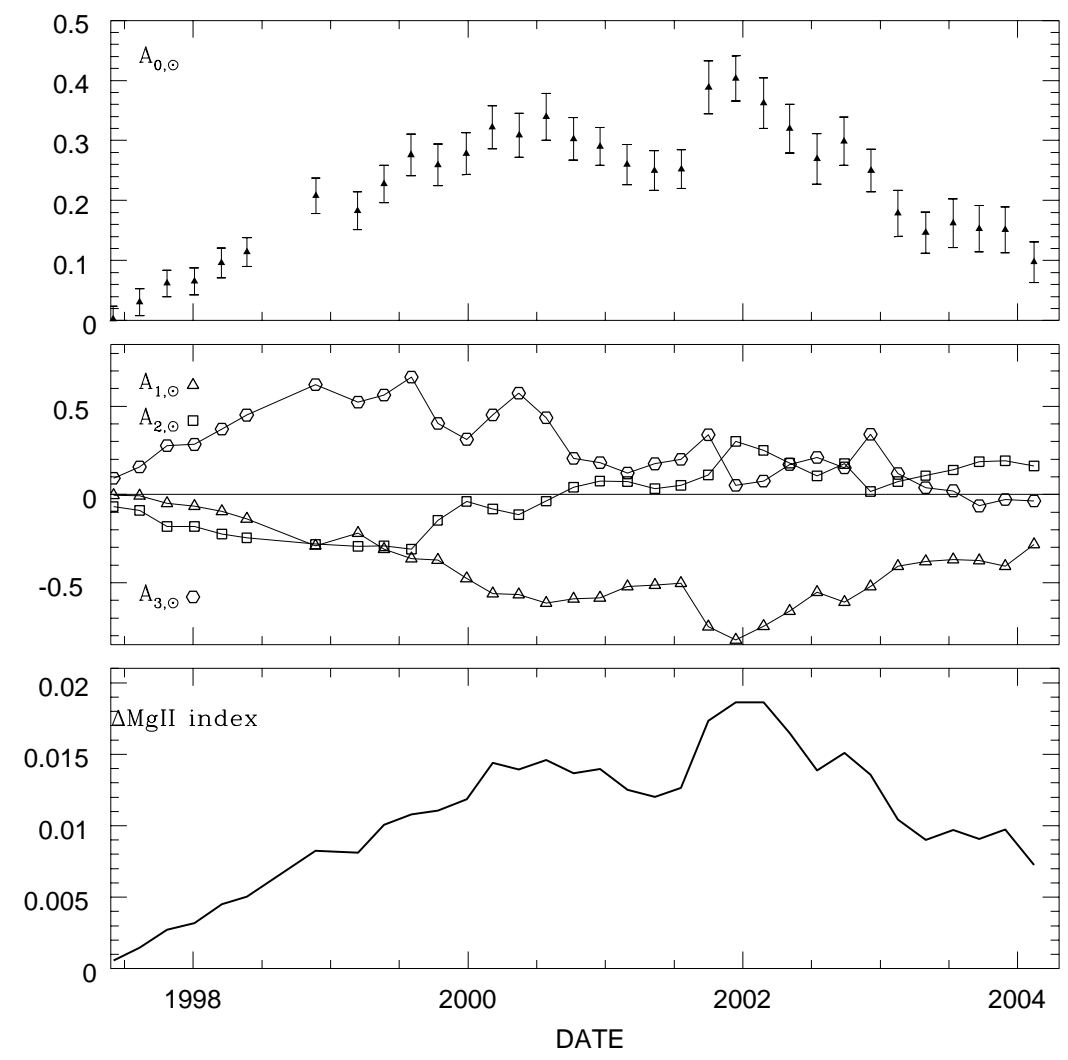

FIGURE 1. Low order $A_{k}$ coefficients describing the source of the oscillation frequency changes and the changes in the MgII activity index during activity cycle 23 . The coefficient $A_{0}$ with a $1 \sigma$ error bar, shown in the top panel, describes the mean-frequencies shifts. The next three coefficients are shown in the middle panel. The errors bars are similar to those of $A_{0}$.

By comparing the top and lower panels in Figure 1 (similar plots are shown in Figure 3 of M07), we see the excellent correlation of the parameter $A_{0}$ with the changes in the $\mathrm{MgII}$ index. Similar correlations exist for the CaII and other activity indexes. More complicated variability patterns of the higher order $A_{k}$, shown in the mid panel of Figure 1, are closely related to the shift of the activity from intermediate to low latitudes. We may see that there is a correlation between $-A_{1}$ and $\Delta i_{\mathrm{MgII}}$ but other coefficients reach their extreme values at different phases of the cycle.

Having determined the first four $A_{k}$-coefficients, we may calculate the frequency shifts for modes with degrees $\ell \leq 3$. Ignoring small changes in the $a_{i}$ coefficients, related to the torsional oscillations, we get from equations (6) and (7)

$$
\Delta v_{n l m}=\frac{R}{M} \sum_{k=0}^{\ell} A_{k} Q_{n \ell}\left(D_{c, k}\right) \kappa_{k, l m}
$$

where $R$ and $M$ are expressed in solar units. We keep these quantities, because we want to apply this equation to other stars.

With known $A_{k}(t)$, equation (10) allows us to evaluate changes in the mode frequencies within individual multiplets of low degrees. The plots in Figure 2 show the calcu- 


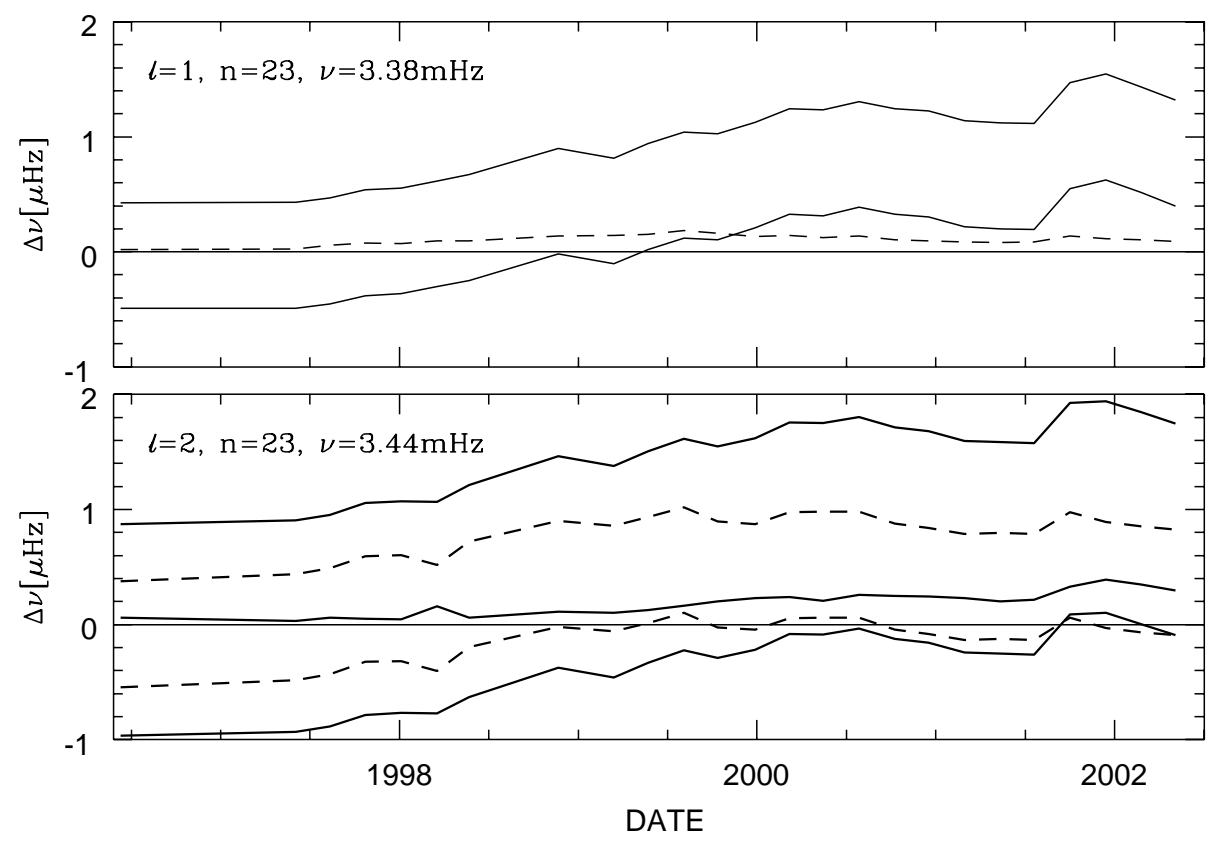

FIGURE 2. The changes in the multiplet structure during the activity cycle 23 for two selected multiplets calculated from the $a_{0}, a_{1}$, and $a_{2}$ SOHO MDI data. The dashed lines refer to modes, which correspond to odd values of $\ell+|m|$ and thus are not detectable in the Sun. There is a drastic departure from symmetry about the zonal $(m=0)$ frequency at high activity.

lated frequency changes during cycle 23 of all components in the two selected multiplets. The plots, which begin at the solar minimum phase, take into account rotational frequency shifts, including the linear shift of $m \times 0.45 \mu \mathrm{Hz}$ and the tiny second-order effect arising from the centrifugal distortion. At the minimum phase, the multiplets are nearly symmetric about the $m=0$ mode frequency. The small asymmetry of the $\ell=2$ multiplet comes mainly from the $a_{4}$ coefficient, which arises from the polar field. Even the smaller asymmetry of the $\ell=1$ multiplet comes solely from the centrifugal distortion. At the solar maximum the asymmetry is very large. However, this spectacular signature of the magnetic activity is not easy to detect. In fact the asymmetry of an $\ell=2$ multiplet was directly measured by Chaplin et al. (2003), long after the first seismic signature of the solar activity has been detected by Woodard and Noyes (1985).

\section{PREDICTIONS FOR OTHER STARS}

The basic assumption in our prediction presented in M07 of the $\ell=0$ mode frequency changes in $\beta$ Hyi was that the relation

$$
A_{0}=22 \Delta i_{\mathrm{MgII}}
$$

which was found for various phases of the solar cycle is valid for other stars. Instead of $i_{\text {MgII }}$, one may use any other global activity index available for the Sun and for a considered star. The richest data on global activity are from the Mount Wilson Observa- 
tory - HK Project, which uses CaII. Unfortunately, this is a northern hemisphere project, which neither includes $\beta$ Hyi, nor any other well studied solar-like pulsators. To apply relation (10), we still had to scale $D_{c, 0}$ and for that we assumed that it is proportional to the pressure scaleheight at the photosphere, that is

$$
D_{c, 0} \propto H_{p} \propto L^{0.25} R^{1.5} M^{-1} .
$$

In order to make prediction for nonradial modes, we have to adopt a scaling of the parameters at $k>0$ in equation (7). The simplest choice is

$$
A_{k} \propto A_{0} \propto \Delta i_{\mathrm{MgII}} \quad \text { and } D_{c, k} \propto D_{c, 0} .
$$

The first proportionality amounts to assume the same butterfly diagram pattern as in the Sun. The second is another extrapolation of the property found in the solar data. Once we make these additional assumptions, we may calculate, with the use of equation (10), the expected frequency shift of low degree modes for any star for which the shift in $i_{\mathrm{MgII}}$, or any other global activity measure, is known.

If we had measurements of the individual mode frequencies within multiplets, we could test our prediction based on equation (10) with single-season observations because the same equation describes the momentary $v(m)$-dependence induced by the momentary magnetic activity. However, such measurements may be difficult, as we learnt from the case of the low- $\ell$ solar oscillation. The problem is that the width of the peaks in the oscillation spectrum is larger than the frequency splitting between the modes. We thus expect that the mean (averaged over multiplet components) frequency shifts will be the first detected seismic signature of the magnetic activity in distant stars.

In order to calculate the mean frequency shifts, we have to weight the individual components which depend on the inclination of the rotation axis to the line of sight, $\theta_{o}$. The weights should be proportional to the power, which -on average- is proportional to $\left|Y_{\ell}^{m}\left(\theta_{o}, 0\right)\right|^{2}$. Hence (see equation 10 ), the mean shifts are given by

$$
\Delta v_{n \ell}=\frac{2 \ell+1}{2} \frac{R}{M} \sum_{k=0}^{\ell}\left[\sum_{m=-\ell}^{m=\ell}\left|Y_{\ell}^{m}\left(\theta_{o}, 0\right)\right|^{2} \kappa_{k, l m}\right] A_{k} Q_{n \ell}\left(D_{c, k}\right) .
$$

An unknown inclination introduces additional uncertainty to our prediction for nonradial modes.

\section{THE CASE OF $\beta$ HYDRI}

The G2IV star $\beta$ Hyi was chosen by M07 for predicting radial mode frequency changes. Modes with $\ell \leq 2$ were measured in the two well separated seasons: 2000.5 (Bedding et al. 2001) and 2005.7 (Bedding et al. 2007). There are eight identified p modes with $\ell \leq 2$ in both data sets. Bedding et al. (2007) find only an insignificant mean frequency shift of $0.1 \pm 0.4 \mu \mathrm{Hz}$ between these dates.

Archival IUE spectra taken between 1978.5 and 1995.5 are available for this star allowing to determine the changes in the MgII index. The new analysis presented in M07 

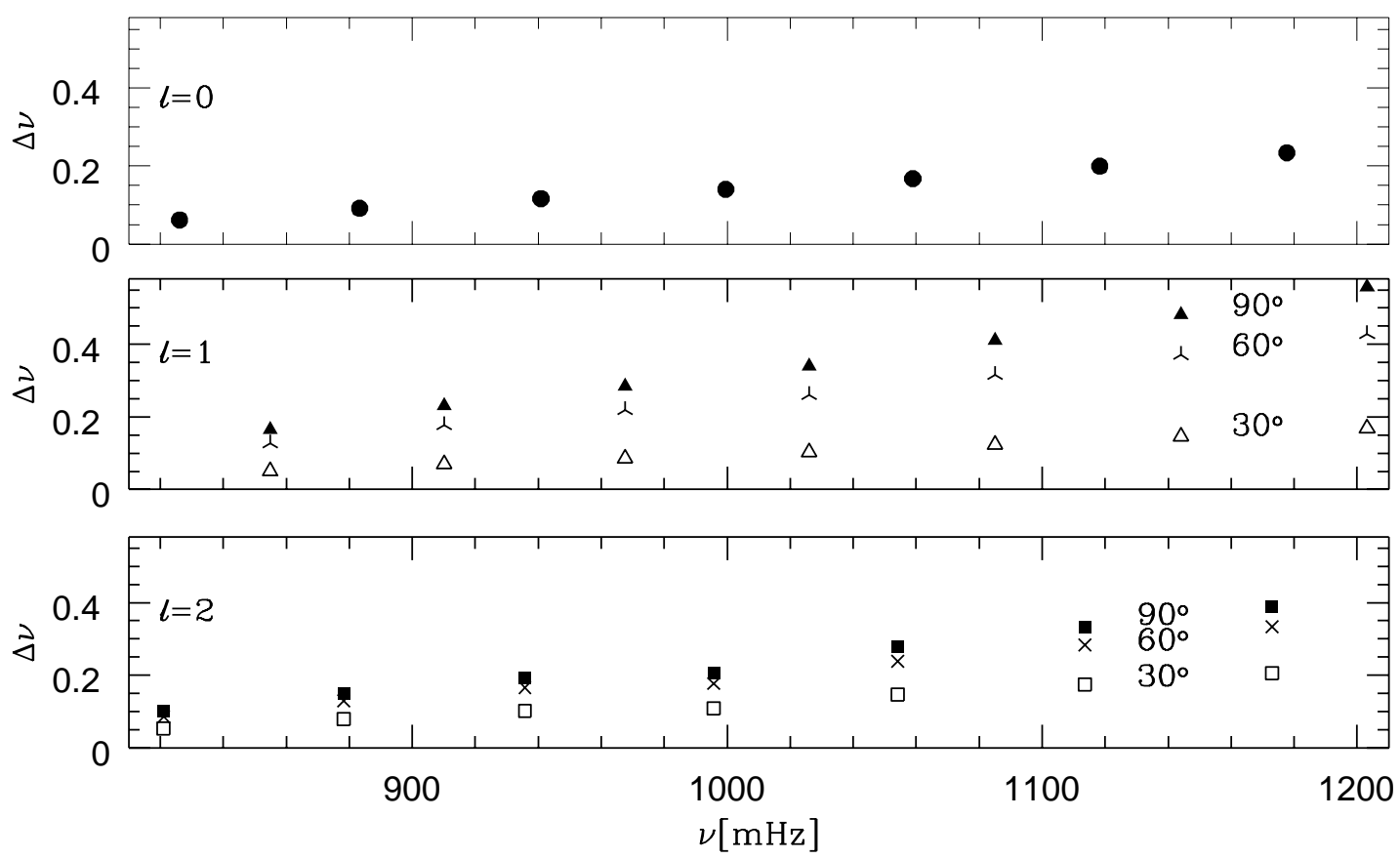

FIGURE 3. Predicted oscillation frequency shifts in $\beta$ Hyi between its activity minimum (2004.5) and maximum (2010.5). The dates were inferred in M07 on the basis of the $i_{\text {MgII }}$ behaviour between 1978.5 and 1995.5

has led to an estimated cycle period of $12 \mathrm{y}$ and to a full amplitude of $\Delta i_{\mathrm{MgII}}=0.15$. Both numbers are not very different from their solar counterparts. The last observed activity minimum took place around 1992.5. In 2000.5 the star was about four years before the next estimated minimum and in 2005.7 about a year after. This means that the interval is not well suited for testing our predictions but there is no better data available on frequencies and activity for any object.

In order to apply equation (13) for predicting frequency shifts, we need a stellar model. The one used by M07 and here has mass, $M=1.1$, radius, $R=1.82$, luminosity, $L=3.17$ (all in solar units), age $=6.7 \mathrm{~Gy}$, and the metallicity parameter $Z=0.0138$. It reproduces the observed mean parameters of the star and the large frequency separation. The values of $A_{k}$ and $D_{c}$ were calculated according to relations (11) and (12), adopting $\Delta i_{\mathrm{MgII}}=0.8 \Delta i_{\mathrm{MgII}, \odot}$ and $D_{c, \odot}=0.3 \mathrm{Mm}$. The numbers calculated for modes identified in the $\beta$ Hyi oscillation spectrum by Bedding et al. (2007) are shown in Figure 3. From the numbers in this figure, we may see that within a few years we have a chance to detect a marginally significant seismic signature of the high activity state of $\beta$ Hyi. However, a major improvement in frequency measurements is needed in order to learn more about the star's activity changes by means of asteroseismology. Once it is achieved and the differences in frequency shifts between modes of different degrees are measured, we may get a useful constraint on the longitudinal dependence of the activity changes. 


\section{DISCUSSION}

The presented method of calculating an expected seismic signature of stellar magnetic activity consists in extrapolating the solar relation between changes in the p-mode frequencies and the chromospheric activity indices. The extrapolation lacks a sound physical justification so it should be regarded as a very uncertain forecast.

Numerical predictions of the frequency shifts were given for $\beta$ Hyi, which, like our Sun. is not a very active star. The star is brighter and more evolved than the Sun but there are no large differences between the two objects. The predicted shifts were found similar to those measured in the Sun. They are small and consistent with the null results of existing measurements but they may be measurable within the next few years.

It could be more interesting to test the prediction for a more active object. Unfortunately, there are none among those stars for which data on solar-like oscillations are available. Magnetic activity inhibits precise frequency measurements. Indeed, the analysis of the data collected during the solar cycle 23 shows that the widths of the peaks in the oscillation spectra increase by some $15 \%$ at the high activity phase (JiménezReyes et al. 2003). On the other hand, since more active stars are also faster rotators, the multiplet structure should be easier to resolve. Thus, I believe such objects should be included in the list of asteroseismic targets. The forecast based on the extrapolation of solar data to more active stars is more risky. However, regardless whether the forecast is right or wrong, its comparison with reality will teach us something interesting about stellar activity.

\section{ACKNOWLEDGMENTS}

This work was supported by the Polish MNiI grant No. 1 P03D 02128.

\section{REFERENCES}

Bachmann K. T., Brown T. M., 1993, ApJ, 411, L45

Baliunas S. L., Donahue R. A., Soon W., Henry G. W., 1998, in Donahue R. A., Bookbinder J. A, eds, ASP Conf. Ser. Vol. 85, Cool Stars, Stellar Systems, and the Sun. Astron. Soc. Pac., San Francisco, p. 153

Bedding T. R. et al., 2001, ApJ, 549, L105

Bedding T. R. et al., 2007, ApJ, 663, 1315

Bedding T. R., Kjeldsen H., 2007, Comm. Asteroseis., 150, 106

Chaplin W. J. et al., 2003, MNRAS, 343, 343

Dziembowski W. A., Goode P. R., 2004, ApJ, 600, 464

Elsworth Y. et al., 1994, ApJ, 434, 801

Goldreich P., Murray N., Willette G., Kumar P., 1991, ApJ, 370, 752

Jiménez-Reyes S. J., García R. A., Jiménez A., Chaplin W. J., 2003, ApJ, 595, 446

Libbrecht K. G., Woodard M. F., 1990, Nat., 345, 779

Metcalfe T. S., Dziembowski W. A., Judge P. G., Snow M., 2007, MNRAS, 379, L16 (M07)

Regulo C., Jimenez A., Palle P. L., Perez Hernandez F., Roca Cortes T., 1994, ApJ, 434, 384

Schou J., Christensen-Dalsgaard, J., Thompson M. J., 1994, ApJ, 433, 389

Woodard M., Noyes R., 1985, Nat., 318, 449 